\title{
Studying Two-Dimensional Zeolites with the Tools of Surface Science: MFI Nanosheets on $\mathrm{Au}(111)$
}

\author{
John D. Kestell ${ }^{1}$, Jian-Qiang Zhong ${ }^{1}$, Meera Shete ${ }^{3}$, Iradwikanari Waluyo ${ }^{2}$, Jerzy T. \\ Sadowski ${ }^{1}$, Dario J. Stacchiola ${ }^{1}$, Michael Tsapatsis ${ }^{3}$, J. Anibal Boscoboinik ${ }^{1 *}$ \\ ${ }^{1}$ Center for Functional Nanomaterials, Brookhaven National Laboratory, Upton, NY \\ 11973, United States. \\ 2 Photon Science Division, National Synchrotron Light Source II, Brookhaven \\ National Laboratory, Upton, NY 11973, United States. \\ ${ }^{3}$ Department of Chemical Engineering, University of Minnesota, United States.
}

*Corresponding author. E-mail address: jboscoboinik@bnl.gov (J. Anibal Boscoboinik)

\begin{abstract}
While surface science has provided fundamental insights into a variety a materials, the most used catalysts in the industry, namely zeolites, still remain a challenge. The recent preparation of two-dimensional versions of MFI zeolite frameworks and the possibility of their deposition on electrically conductive supports provides for the first time a viable strategy to perform detailed studies on industrially relevant zeolites using the vast toolkit of surface science. In this work we demonstrate the use of infrared reflection absorption spectroscopy (IRRAS) and synchrotron-based x-ray photoelectron spectroscopy (XPS) to study these materials. Furthermore, polarization modulation IRRAS is used to study the adsorption of methanol and its effect in phonon vibrations of the zeolite framework. The possibility of using surface science methods, in particular under ambient pressure conditions, for the study of well-defined zeolites and other microporous structures opens new avenues to understand structural and mechanistic aspects of these materials as catalysts, adsorbents and molecular sieves.
\end{abstract}

\section{Keywords}

Zeolites, Surface Science, X-ray Photoelectron Spectroscopy, Infrared Reflection Absorption Spectroscopy,

\section{Introduction}

Zeolites are fascinating materials from the topological point[1] of view and of critical importance in several industrial processes related to separation of molecules, adsorption and catalysis.[2, 3] However, their 3D structures impose certain limitations 
to interrogate them with atomic precision and their detailed fundamental study using surface science methods has been very limited due to technical reasons.[4] One of the most important burdens is the fact that several of the surface science methods either require or benefit from the material under study being electrically conductive, which zeolites are not. Recent work has shown that crystalline two-dimensional aluminosilicate frameworks consisting of a planar arrangement hexagonal prisms, also known as double six-membered rings (D6R), as secondary building blocks, show similar chemical behavior as zeolites and can be used for model system studies.[5] This structure however does not contain the wide range of 3D pores and channels present in 3D zeolites and while crucial information can be obtained from them and their interaction with molecules, they have important limitations, which have been outlined in a recent perspective article.[4]

The recent development of thin zeolite nanosheets [6-9] containing a full range of $3 \mathrm{D}$ pores and channels and their deposition on conductive supports [10] provides now the opportunity to use surface science methods such as X-ray Photoelectron Spectroscopy (XPS), Scanning Tunneling Microscopy (STM) and Infrared Reflection Absorption Spectroscopy (IRRAS) to study these materials with unprecedented atomic level detail.[4] Furthermore, while surface science experiments are often performed under ultra-high vacuum conditions (UHV), the advent of elevated pressure versions of surface science methods in the last few years, allows carrying out these studies at conditions of pressure and temperature more relevant to the practical application of zeolites.

In this paper we explore these new opportunities by using Polarization Modulation IRRAS (PM-IRRAS) and Ambient Pressure XPS (AP-XPS) to study the case of all-Si MFI zeolite (or silicalite) nanosheets deposited on an $\mathrm{Au}(111)$ substrate. We chose to use atomically flat gold as a substrate because of its inert nature, to avoid any potential reactions of methanol on more reactive substrates, and the (111) face since it is as well the thermodynamically most stable one. Additionally, $\mathrm{Au}(111)$ remains clean upon air exposure. Note that MFI is the framework of ZSM-5, one of the most important zeolites for catalysis applications, including the methanol to gasoline conversion [11] among many others. XPS provides information on the composition of the structure as well as the oxidation states of all the elements in the framework and potentially adsorbates within the pores of the framework. IRRAS on the other hand, provides exquisite information on framework phonon vibrations as well as vibrational modes of molecules adsorbed or trapped within the pores. In order to illustrate the use of this technique, we study the case of methanol adsorption on MFI nanosheets, of importance to applications in catalysis for methanol to hydrocarbon conversion [11, 12] and for separation of alcohols from aqueous solutions.[13-16] 
While this work provides a proof-of-concept of the surface science approach by IRRAS and XPS, we see no reason for other surface science methods not to be applied to the study of this and other two-dimensional nanoporous materials and we expect that there will be substantial growth in this direction in the coming years, including the application of scanning probe microscopies to perhaps even atomically resolve the surface of these structures.

\section{Materials and methods}

Sample Preparation. The $\mathrm{Au}(111)$ single crystal surface was cleaned with cycles of $\mathrm{Ar}^{+}$sputtering and annealing at $900 \mathrm{~K}$. The $3 \mathrm{~nm}$ thick MFI nanosheets were then deposited onto the $\mathrm{Au}(111)$ surface using a Langmuir-Schaefer method described in reference [10]. Studies on the structure and integrity of the MFI nanosheets prepared and deposited following the same procedure used for this work were reported in previous work, using high-resolution TEM and electron diffraction. [10] Further crystallographic investigation on the structural integrity and thickness of the nanosheets was performed by diffraction tilting experiments in the TEM. [17] After deposition on $\mathrm{Au}(111)$, the sample was heated to $775 \mathrm{~K}$ in a $20 \mathrm{cc} / \mathrm{min}$ flow of $\mathrm{O}_{2}$ at 1 Atm pressure in a tube furnace to remove carbonaceous contamination from the

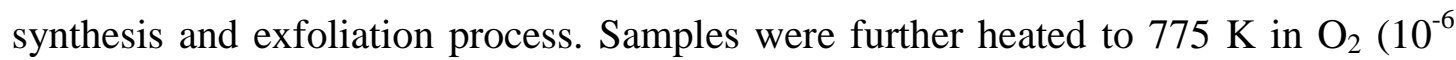
mbar) inside the instruments to remove carbonaceous species that might have adsorbed during air transport into the vacuum chambers. Note that XPS showed a significant amount of carbon on the surface after exposure to air and introduction in the UHV chamber. These carbonaceous species were however easily removed by annealing in low $\mathrm{O}_{2}$ pressures, as evident from XPS data.

PM-IRRAS experiments were carried out at the Center for Functional Nanomaterials at BNL in a home-built setup described in detail in ref. [18] Briefly, this instrument is equipped with a Bruker Vertex 70 spectrometer and is capable of PM-IRRAS experiments at pressures up to $1 \mathrm{Atm}$. The beam is reflected from the surface plane at an $8^{\circ}$ angle and the signal measured with a liquid-nitrogen-cooled MCT detector. The 2.75" spherical cube chamber has $\mathrm{BaF}_{2}$ windows to allow IR light to enter and exit the chamber and to withstand the potentially high pressures the instrument is capable of. $A P$-XPS measurements were carried out at the CSX-2 Beamline of the National Synchrotron Light Source II (NSLS-II). [19] The main chamber (base pressure $2 \times 10^{-9}$ mbar) of the end-station was equipped with a differentially pumped hemispherical analyzer (Specs Phoibos 150 NAP), which was offset by $70^{\circ}$ from the incident synchrotron light and $20^{\circ}$ from the surface normal of the sample. A photon energy of $750 \mathrm{eV}$ was used in our studies, which was calibrated and referenced to the Fermi level of the $\mathrm{Au}(111)$ substrate. The $\mathrm{Au}(111)$ crystal was mounted on a ceramic button 
heater and the temperature was monitored through a type $\mathrm{K}$ thermocouple underneath the crystal.

\section{Results and discussion}

Crystalline materials, such as zeolites show phonon framework vibrations detectable within the mid-IR range, i.e.: $4000-400 \mathrm{~cm}^{-1}$.[20] These phonon vibrations are associated to $\mathrm{T}-\mathrm{O}$ stretching, where $\mathrm{T}$ is a tetrahedral atom, most commonly $\mathrm{Si}$, as in the case described here. These phonon modes are found below $1300 \mathrm{~cm}^{-1}$ and extend beyond the mid-IR range into the far-IR. Representations of the top and side views of the MFI nanosheet are shown in Figure 1.

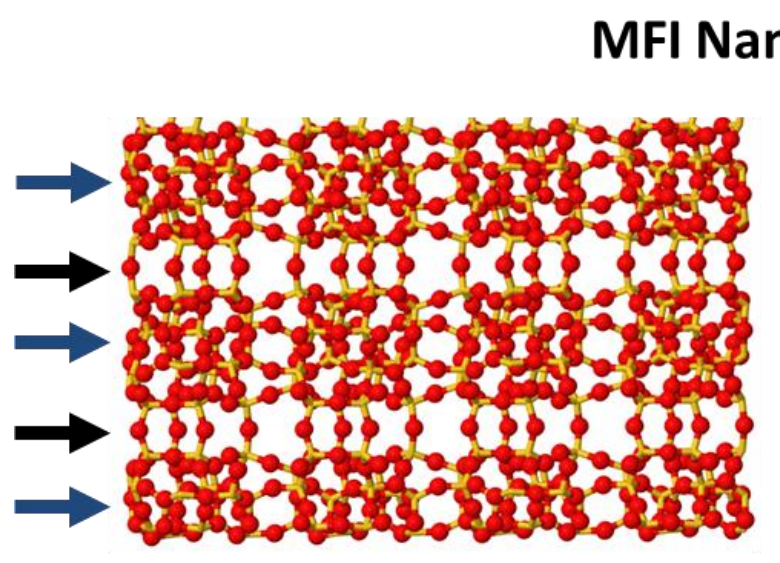

Side View

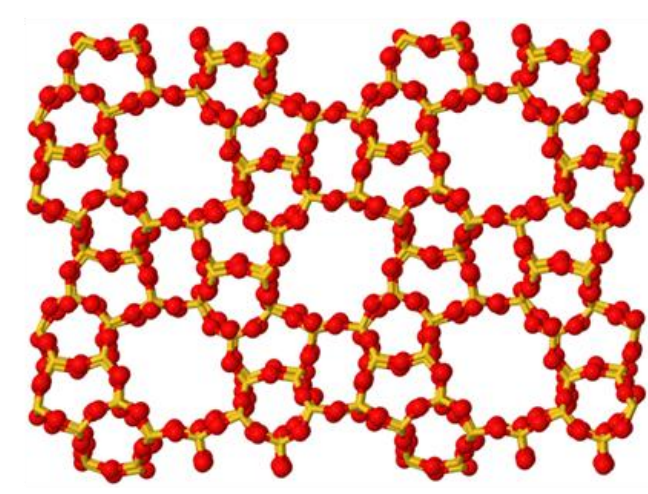

Top View

Figure 1. Side and top view of a structural representation of the MFI nanosheets.

Figure 2 shows an AFM image of the MFI nanosheets after deposition, where coverages between one and two monolayers are evident in the image. Uncovered regions as well as regions with more than two layers are also observed in a smaller proportion. Brighter areas could be related to remaining carbonaceous species, which are known to be present on the surface as it will be described later. MFI nanosheets have mostly rectangular shapes with sides of lengths in the order of hundreds of nanometers. 


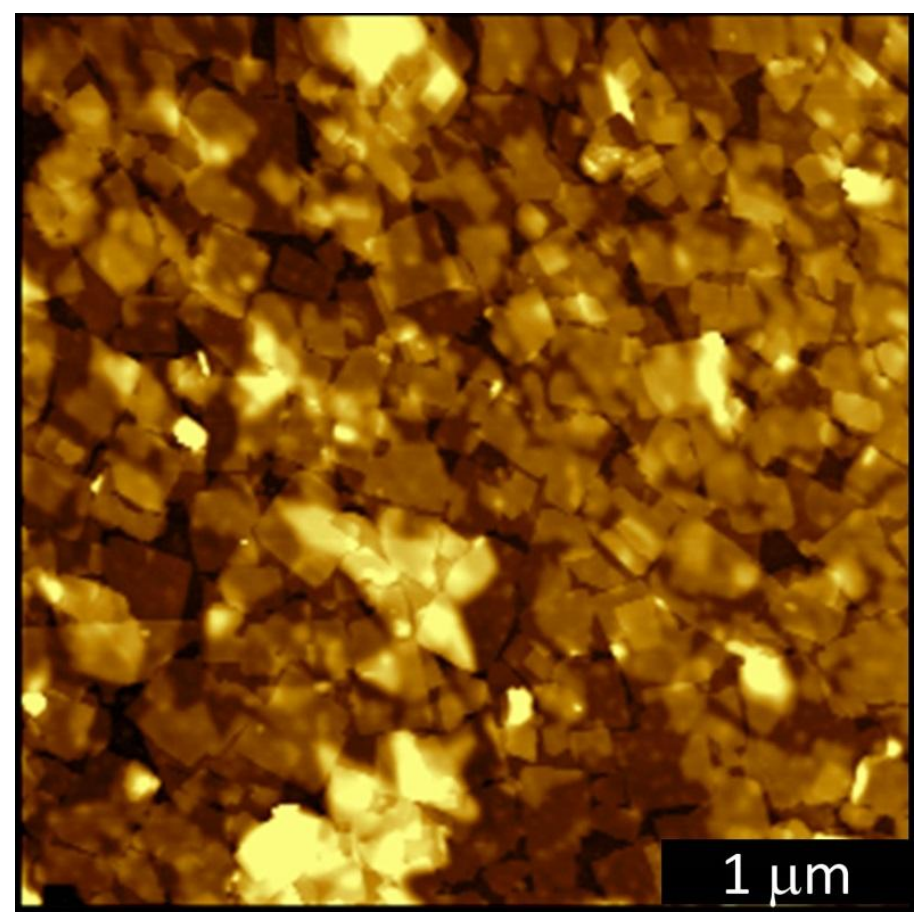

Figure 2. AFM image taken in air after deposition of MFI nanosheets on $A u(111)$ using a Langmuir-Schaefer method. [10]

IRRA spectra of all-Si MFI nanosheets on $\mathrm{Au}(111)$ taken at ultra-high vacuum conditions (black line in Figure 3) show two main framework phonon vibrations, a high frequency (HF) mode at $1246 \mathrm{~cm}^{-1}$ and a low frequency (LF) mode at $1186 \mathrm{~cm}^{-1}$. Note that these values are higher than previous reports for bulk all-Si MFI (or silicalite-1) diluted in $\mathrm{KBr}$, showing these two phonon modes at lower frequencies $1230 \mathrm{~cm}^{-1}$ and $1115 \mathrm{~cm}^{-1}$ using diffuse reflectance IR.[21] A different study shows these two phonons at $1240 \mathrm{~cm}^{-1}$ and $1129 \mathrm{~cm}^{-1}$.[22] Work by Farmer and Russell on layered silicates showed a strong dependence of phonon frequency with size of the crystallites, with the frequency increasing for smaller crystals.[23] This is consistent with our observation of higher frequencies for 2D-MFI as compared to 3D-MFI.

Aside from the lower frequencies, in these previous reports the HF band is lower in intensity than the LF band. In our case the HF band is higher in intensity than the LF band. It is expected in fact that, since the MFI nanosheet is a two-dimensional system, these phonons will differ from those of bulk MFI. Additionally, due to the selection rules of IRRAS on a metallic support, modes normal to the surface will be enhanced in intensity and modes parallel to the surface plane will not be observed. Thus, the fact that the HF band at $1246 \mathrm{~cm}^{-1}$ is more intense in the 2D zeolite in relative terms to its counterpart for bulk MFI indicates that it is associated with modes normal to the $\mathrm{Au}(111)$ surface plane. Previous assignments in the literature for these phonons are not very clear and further studies are necessary to obtain a more detailed understanding of what parts of the framework they are associated with. Zecchina et al. 
assign these modes to $A$ and $B$ components of the triply degenerated $T_{2}$ mode, resulting from the broken symmetry of the $\mathrm{SiO}_{4}$ building units within the zeolite structure. [21] However, methanol adsorption results presented below suggest that these two peaks, $1246 \mathrm{~cm}^{-1}$ and $1186 \mathrm{~cm}^{-1}$, may be associated with different parts of the framework, as discussed below.

The interaction of methanol with the 2D-MFI framework was studied from high vacuum conditions, at which no methanol is evident in the framework, and up to 16 Torr. The corresponding PM-IRRA spectra are shown in figure 3. The spectrum at 16 Torr methanol is shown with a blue solid line. The methanol related peaks can be clearly seen in the $1000 \mathrm{~cm}^{-1}$ to $1100 \mathrm{~cm}^{-1}$ region, with a main sharp peak at 1034 $\mathrm{cm}^{-1}$ and two broad bands at $\sim 1060 \mathrm{~cm}^{-1}$ and $1015 \mathrm{~cm}^{-1}$. A reference spectrum of liquid methanol shows a single peak in this region,[24] at $1022 \mathrm{~cm}^{-1}$ from the CO stretching vibration. At this pressure, the $1186 \mathrm{~cm}^{-1}$ peak shifts to $1176 \mathrm{~cm}^{-1}$, while no changes in frequency are observed on the $1246 \mathrm{~cm}^{-1}$ peak, suggesting that the LF peak is related to the region of the structure with which adsorbed methanol molecules interact. After evacuation of methanol the LF peak shifts back to its original position at $1186 \mathrm{~cm}^{-1}$, shown with a red dashed line in Figure 3, indicating that the methanol adsorption is reversible, as expected. From previous calculations in the literature,[22] it is assumed that methanol adsorbs within the pores, and thus the $1186 \mathrm{~cm}^{-1}$ phonon should be associated with these pores. Conversely, the unaffected phonon at 1246 $\mathrm{cm}^{-1}$ is likely related to the $\mathrm{Si}-\mathrm{O}-\mathrm{Si}$ linkages between the pores. We tentatively suggest that this mode is related to the $\mathrm{Si}-\mathrm{O}-\mathrm{Si}$ linkages shown by black arrows in figure 1. It is worth emphasizing at this point that this is an Al-free MFI framework, and thus there are no bridging $\mathrm{OH}$ groups with which methanol would interact more strongly. However, the interaction with silanols groups resulting from defects, especially at the surface of the nanosheets cannot be discarded. Evidence of their presence is discussed below, in relation to the $956 \mathrm{~cm}^{-1}$ band observed in the spectra. Bleken el al. have carried out FTIR experiments on Al-containing MFI nanosheets, or H-ZSM-5, where they explore the interaction of different molecules with $\mathrm{OH}$ groups present in the structure. [25] Ryoo et al. did a full characterization of MFI nanosheets, including IR data showing that Brönsted acid sites are present on the surface of the nanosheets for the Al-containing form, but without being able to look into lower wavelengths where the $\mathrm{Si}-\mathrm{O}$ phonon related features are observed. [26] 


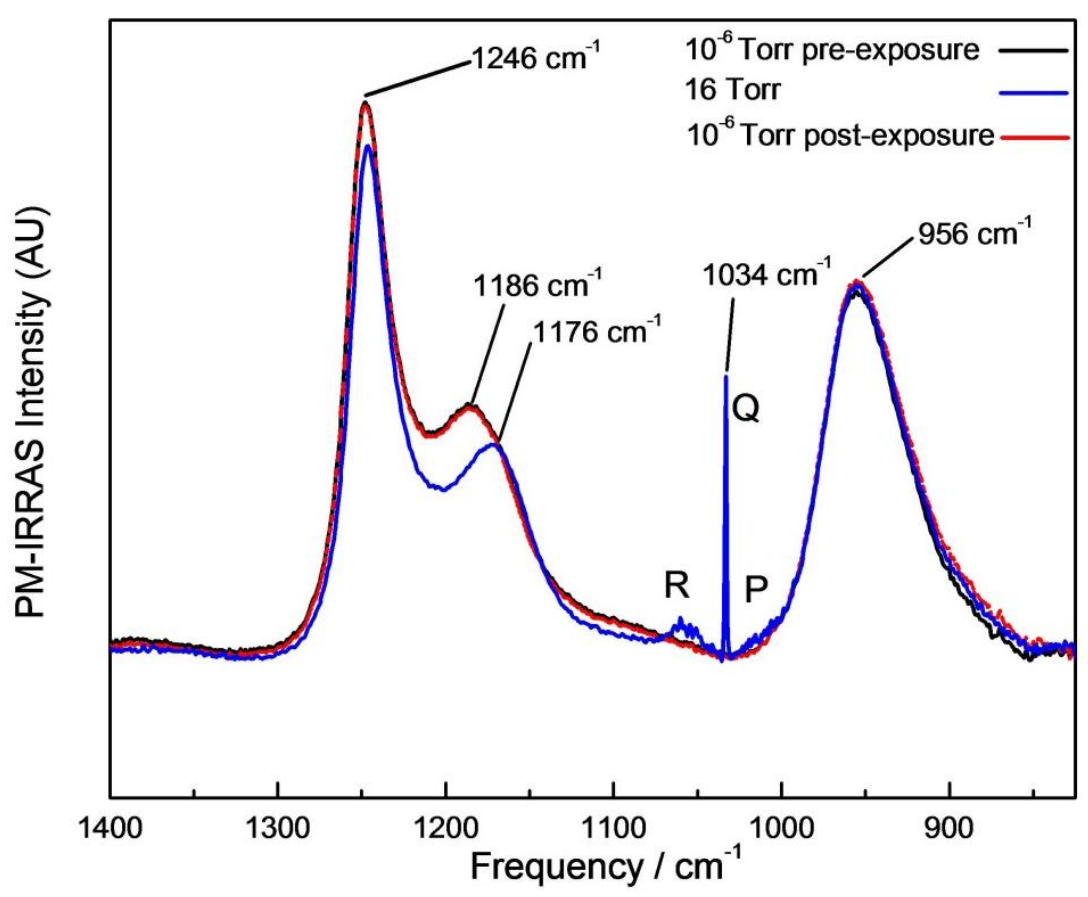

Figure 3. PM-IRRAS of methanol on 2D-MFI/Au(111) at high vacuum condition and under 16 Torr of methanol.

The main methanol peak observed at $1034 \mathrm{~cm}^{-1}$ under a pressure of 16 Torr is close to the $\mathrm{C}-\mathrm{O}$ stretch for gas phase, which appears at $1030 \mathrm{~cm}^{-1}$. [27] However, the $\mathrm{P}$ and $\mathrm{R}$ branches from a typical rotovibrational spectrum of gas phase methanol are greatly attenuated, which we attribute to confinement of methanol within the pores. While it is tempting to assign these peaks to incomplete background subtraction or detector saturation, it should be noted that at methanol pressures of 20 Torr on clean gold, these peaks are not observed. Since the R and P branches of the PM-IRRAS spectra are greatly attenuated with respect to the $\mathrm{Q}$ branch, a more likely explanation is that the rotational freedom of methanol molecules (inside the pores) is drastically reduced, as would be expected and consistent with direct interaction of the molecule with the pore as described above. The attenuation and shift of the LF phonon mode is likely the result of vibrational coupling of the $\mathrm{Si}-\mathrm{O}-\mathrm{Si}$ and $\mathrm{C}-\mathrm{O}$ modes, which are in close proximity, both energetically and spatially. To further support this statement we compared the PM-IRRAS signal where the surface species are seen to a spectrum taken without using the photoelastic modulator, at 12 Torr, where the gas phase signal dominates the spectrum. This is shown in Figure S1 of the supporting information, where the difference can be clearly seen, both in the intensity of the $\mathrm{P}$ and $\mathrm{R}$ branches and in the $4 \mathrm{~cm}^{-1}$ shift from $1030 \mathrm{~cm}^{-1}$ in gas phase to $1034 \mathrm{~cm}^{-1}$ for methanol trapped in the pores.

Figure 4 shows spectra in vacuum and at increasing methanol pressures, from 0.1 Torr to 16 Torr. The effect of gradual changes in methanol pressure in the LF phonon 
vibration is evident from these spectra. It is interesting to note that while the 1034 $\mathrm{cm}^{-1}$ peak is not visible at pressures below 5 Torr, the shift and attenuation of the phonon peak persists down to $\sim 0.1$ Torr. This indicates that methanol is already present within the framework and has a strong effect in the LF phonon mode, even when the amount is low enough that the $\mathrm{CO}$ stretch is not observed in the IR spectrum. It is worth emphasizing here that the intensity of the peaks cannot be directly correlated to the concentration of molecules, as it strongly depends on the orientation of the dynamic dipole that gives rise to the absorption of IR light. In fact this is clear for this case in which the presence of methanol is evident by the LF phonon shift even before the $\mathrm{C}-\mathrm{O}$ stretching vibration is visible in the spectrum, suggesting that the $\mathrm{C}-\mathrm{O}$ bond is oriented parallel to the surface plane for low coverages. Isotherms for methanol adsorption on bulk silicalite at $303 \mathrm{~K}$ have been published by Dubinin et al.[16] and later simulated by Monte Carlo methods, [28-30] obtaining good agreement between experiment and simulation at elevated pressures. The experimental work shows a slow increase in the concentration of methanol from $\sim 1$ molecule per unit cell 0.01 Torr to $\sim 3$ molecules per unit cell at 3 Torr. The Monte Carlo simulations underestimate the methanol adsorption at these low pressures and they attribute this to the presence of defects in the structure in the experimental case, which are absent in the simulations.[28-30] A rapid increase in methanol adsorption starts at pressures higher than $\sim 6$ Torr. This agrees well with our PM-IRRAS data in which the initial adsorption of low concentrations of methanol already at 0.1 Torr is evident by the shift in the LF phonon mode. 


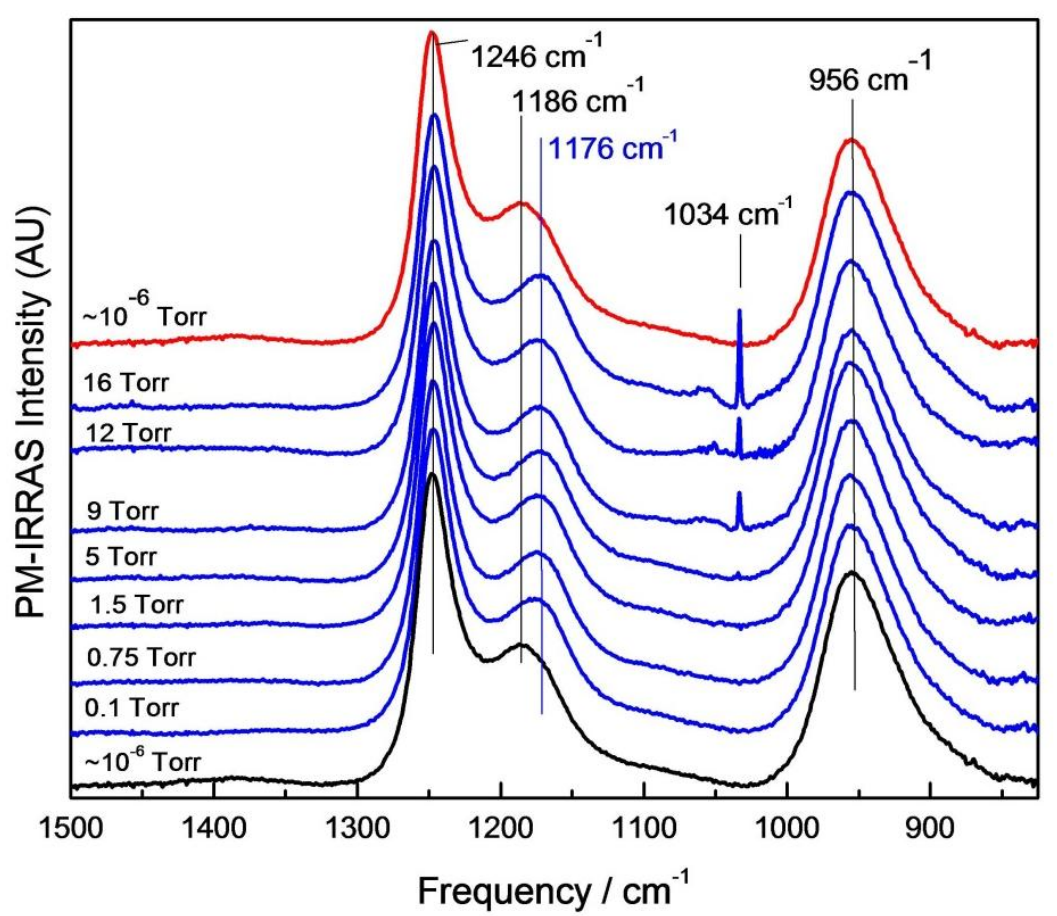

Figure 4. PM-IRRAS of methanol on 2D-MFI/Au(111) at high vacuum and increasing pressures of methanol between 0.1 Torr and 16 Torr.

All spectra in Figure 3 and Figure 4 show also another prominent feature at $956 \mathrm{~cm}^{-1}$. This broad feature has been related to the $\mathrm{Si}-\mathrm{O}$ stretching vibration of silanol groups and $\mathrm{Si}-\mathrm{O}^{-}$groups, [31] and it was also observed for silicalite particles by Zecchina et al. [21] This is in agreement with our experience in this series of experiments, in which we observed that the intensity of this band increases with the aging of the sample. The intensity of this feature was negligible immediately after preparation but grew in intensity after months of exposure to air. In fact, experiments by Ravishankar et al. carried out in Silicalite-1 nanocrystals,[32] clearly show the $\mathrm{Si}-\mathrm{OH}$ vibration at $960 \mathrm{~cm}^{-1}$, which disappears after calcination of the sample. We have verified however that the presence of these silanol groups, most likely at the surface of the nanosheet, does not have an effect in the adsorption of methanol, which shows similar behavior in terms of shifting the LF phonon to lower frequencies, regardless of the intensity of the $956 \mathrm{~cm}^{-1}$ band.

Another technique that can be very useful for the study of zeolites and related materials is X-ray photoelectron spectroscopy (XPS), in particular when synchrotron radiation is used. Figure 5 shows XPS spectra of the MFI nanosheets on $\mathrm{Au}(111)$ for the $\mathrm{O} 1 \mathrm{~s}$ (Figure 5a), Si 2p (Figure 5b) and Au 4f (inset in Figure 5c) core levels as well as for the valence band (Figure $5 \mathrm{c}$ ). The spectra for each core level are taken after exposure to 1 mbar of $\mathrm{O}_{2}$ at $475 \mathrm{~K}$ (purple solid line) and after exposure to $\mathrm{H}_{2}$ at 0.1 mbar at $435 \mathrm{~K}$ (green solid line). The first $\mathrm{O}_{2}$ treatment is carried out in order to remove carbon-containing species adsorbed by the MFI during exposure to air. While 
the initial treatment at $775 \mathrm{~K}$ in a $20 \mathrm{cc} / \mathrm{min}$ flow of $\mathrm{O}_{2}$ at 1 Atm pressure in a tube furnace removes the carbonaceous contamination from the synthesis and exfoliation process, exposure to air results in the adsorption of more weakly bound carbonaceous species, as evident from XPS (data not shown). Upon introducing the sample back in the UHV chamber for the XPS experiments, the milder treatment of $1 \mathrm{mbar}_{2}$ at 475 $\mathrm{K}$ is enough to remove the weakly adsorbed carbon-containing molecules from the MFI nanosheets. The peak at $\sim 11.6 \mathrm{eV}$ in the valence band region can be assigned to the hybridized O 2p-Si 3s, 3p bonding orbitals in Si-O-Si building blocks. [33] Upon exposure to $0.1 \mathrm{mbar}_{2} \mathrm{H}_{2}$ at $435 \mathrm{~K}$ all core levels of the MFI framework shift 0.3 $\mathrm{eV}$ to higher $\mathrm{BE}$, while the $\mathrm{Au} 4 \mathrm{f}_{7 / 2}$ peak remains at $84 \mathrm{eV}$. A similar behavior was observed by Loffler et al. for bilayer $\mathrm{SiO}_{2}$ films on $\mathrm{Ru}(0001)$ where all core levels of the film (but not from $\mathrm{Ru}$ ) shift to higher binding energies when the amount of chemisorbed $\mathrm{O}$ on the $\mathrm{Ru}(0001)$ is reduced.[34] An explanation for these shifts was recently given based on the balance of dipole moments induced by charge transfer from $\mathrm{Ru}(0001)$ to chemisorbed oxygen and from the silica framework to the $\mathrm{Ru}(0001)$ surface. [35] A similar mechanism is probably responsible for the shifts induced here, where the interactions between the MFI framework and the $\mathrm{Au}(111)$ surface may be associated with charge-transfer between the components of the heterostructure. On the $\mathrm{O} 1 \mathrm{~s}$ core level, aside from the main peak at $533.0 \mathrm{eV}$ (shifted to $533.3 \mathrm{eV}$ after $\mathrm{H}_{2}$ treatment) there is a broad shoulder that extends from the main O1s peak down to $529 \mathrm{eV}$. We believe this could be related to framework oxygen atoms at the interface between the MFI nanosheet and the $\mathrm{Au}(111)$ surface. Note that the surface structure of the MFI nanosheet is not well-understood and that dangling bonds are formed upon exfoliation of the single MFI layers which could result in reactive $\mathrm{O}$ at the nanosheet surface, possibly giving rise to the broad shoulder mentioned above. Further studies are needed to elucidate the nature of the MFI surface both at the MFI/Au(111) and MFI/vacuum interfaces. 
(a)

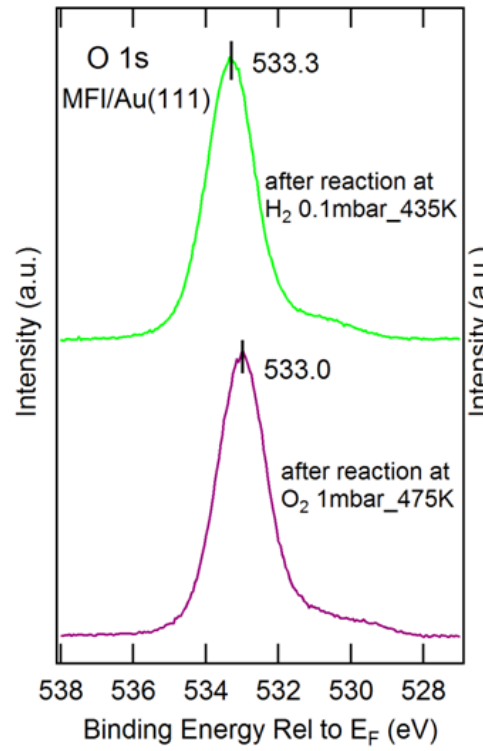

(b)

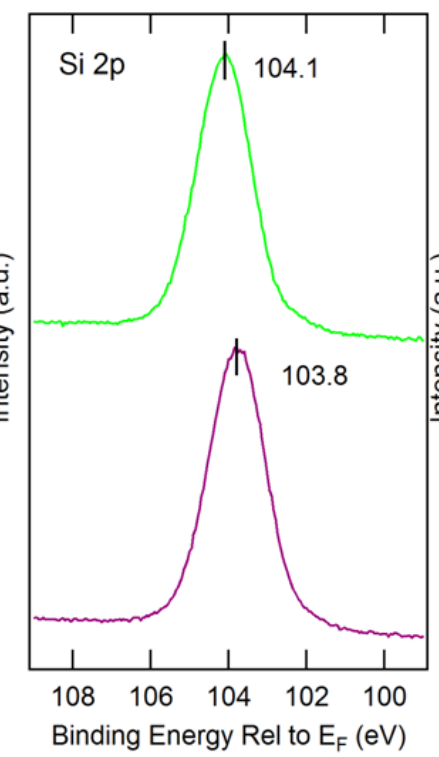

(c)

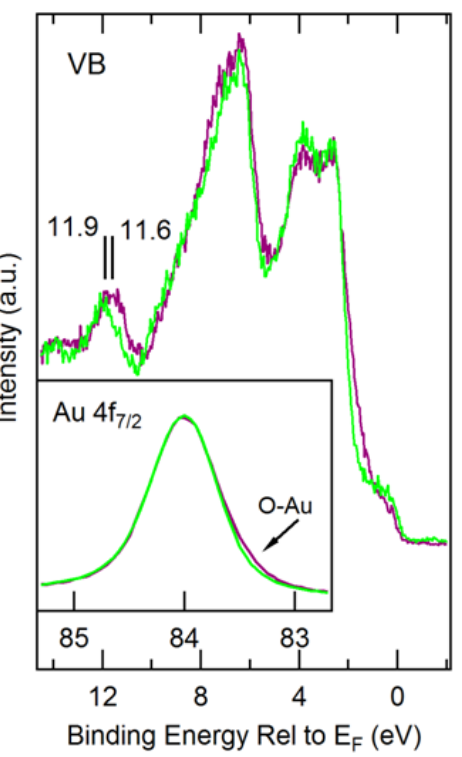

Figure 5. Oxidation and reduction of the MFI nano sheets on the Au(111) surface. The XPS core level spectra of (a) O 1s and (b) Si 2p and (c) valence band were taken in the UHV after the reduction and oxidation reactions as indicated in the figures. The inset in (c) shows the Au $4 \mathrm{f}$ core level spectra. The photon energy is $750 \mathrm{eV}$.

A study on the oxidation and reduction of $\mathrm{Ru}(0001)$ covered by ultra-thin silicate and aluminosilicate crystalline films, including MFI nanosheets, was carried out recently, to explore the influence of nanoscale porosity of a coating layer on its ability to protect the $\mathrm{Ru}(0001)$ surface from oxidation.[19] The aforementioned shifts upon exposure to oxidizing and reducing conditions are also observed in the $\mathrm{MFI} / \mathrm{Ru}(0001)$ case.

The Si 2p core level has a binding energy of $103.8 \mathrm{eV}$ after $\mathrm{O}_{2}$ exposure and 104.1 after reduction with $\mathrm{H}_{2}$, consistent in both cases with a fully oxidized $\mathrm{SiO}_{2}$. Absolutely no elemental $\mathrm{Si}$ is observed which would have a binding energy at $~ 99.9$ $\mathrm{eV}$ if it was present.

The two spectroscopies mentioned in this work can be used for pressures ranging from very controlled ultra-high vacuum conditions up to a few Torr (with AP-XPS) to obtain information at conditions more relevant to the actual application of zeolites. In the case of PM-IRRAS, pressures up to 1 Atm can be used. The data presented here gives just a hint of the potential for the surface science approach to gain detailed knowledge about the chemistry of zeolite materials using 2D-nanosheets as zeolite models. Additionally, structural information can be gained from microscopies such as low energy electron microscopy (LEEM) and scanning probe microscopies, including high resolution scanning tunneling and atomic force microscopies. Furthermore, the example here was of the simple case of all-Si MFI, but Al-containing MFI, ie: ZSM-5 could be used, as well as nanosheets of other relevant framework types. 


\section{Conclusions}

Recent developments in the preparation of two-dimensional zeolites and their deposition onto well-defined conductive single crystal surfaces opens up the possibility of using the wide variety of tools developed through decades by the surface science community for their detailed study. This will result in much deeper levels of understanding of zeolites as well as their surfaces from the structural and chemical points of view. In this work we do a first approach to this idea by using infrared reflection absorption spectroscopy (IRRAS) and X-ray photoelectron spectroscopy (XPS) to study a 3nm thick MFI nanosheet on a $\mathrm{Au}(111)$ single crystal surface. Framework phonon vibrations are evident by IRRAS, although the intensity of the features differs from previous reports on 3D-zeolites. Furthermore, the influence of the adsorption of methanol on the framework phonons is followed by polarization-modulation IRRAS, showing good agreement with experimental methanol adsorption isotherms from the literature. In addition, XPS allows verifying the oxidation state of the elements composing the framework, in this case $\mathrm{Si}$ and $\mathrm{O}$ by looking at their $1 \mathrm{~s}$ core levels. The present work serves to hint at the numerous possibilities ahead of us for the study of zeolites and related porous materials with a vast set of new tools, and the need and opportunity of correlating these experimental studies with theoretical calculations to be able to make definitive assignments of complex 3D zeolite structures.

\section{Acknowledgements}

Research carried out in part at the Center for Functional Nanomaterials and at the CSX-2 beamline of the National Synchrotron Light Source II, Brookhaven National Laboratory, which is supported by the U.S. Department of Energy, Office of Basic Energy Sciences, under Contract No. DE-SC0012704. MFI nanosheet preparation was supported from the Center for Gas Separations Relevant to Clean Energy Technologies, an Energy Frontier Research Center funded by the US Department of Energy, Office of Science, Basic Energy Sciences under Award DE-SC000105. J.Q Zhong is supported by BNL LDRD Project No. 15-010. We thank W.T. Tysoe, T. Kim, N. Akter, M. Wang and D. Lu for useful discussions.

\section{References.}

[1] C. Baerlocher, L.B. McCusker, Database of zeolite structures, www.iza-structure.org/databases2007.

[2] C.C.H. Lin, K.A. Dambrowitz, S.M. Kuznicki, Evolving applications of zeolite molecular sieves, The Canadian Journal of Chemical Engineering, 90 (2012) 207-216. 
[3] B. Yilmaz, U. Müller, Catalytic Applications of Zeolites in Chemical Industry, Topics in Catalysis, 52 (2009) 888-895.

[4] J.A. Boscoboinik, S. Shaikhutdinov, Exploring Zeolite Chemistry with the Tools of Surface Science: Challenges, Opportunities, and Limitations, Catalysis Letters, 144 (2014) 1987-1995.

[5] J.A. Boscoboinik, X. Yu, B. Yang, F.D. Fischer, R. Włodarczyk, M. Sierka, S. Shaikhutdinov, J. Sauer, H.-J. Freund, Modeling Zeolites with Metal-Supported Two-Dimensional Aluminosilicate Films, Angew. Chem. Int. Ed., 51 (2012) 6005-6008.

[6] K. Varoon, X.Y. Zhang, B. Elyassi, D.D. Brewer, M. Gettel, S. Kumar, J.A. Lee, S. Maheshwari, A. Mittal, C.Y. Sung, M. Cococcioni, L.F. Francis, A.V. McCormick, K.A. Mkhoyan, M. Tsapatsis, Dispersible Exfoliated Zeolite Nanosheets and Their Application as a Selective Membrane, Science, 334 (2011) 72-75.

[7] M. Tsapatsis, 2-dimensional zeolites, AIChE Journal, 60 (2014) 2374-2381.

[8] W.J. Roth, P. Nachtigall, R.E. Morris, J. Čejka, Two-Dimensional Zeolites: Current Status and Perspectives, Chemical Reviews, 114 (2014) 4807-4837.

[9] K.V. Agrawal, B. Topuz, Z. Jiang, K. Nguenkam, B. Elyassi, L.F. Francis, M. Tsapatsis, M. Navarro, Solution-processable exfoliated zeolite nanosheets purified by density gradient centrifugation, AIChE Journal, 59 (2013) 3458-3467.

[10] N. Rangnekar, M. Shete, K.V. Agrawal, B. Topuz, P. Kumar, Q. Guo, I. Ismail, A. Alyoubi, S. Basahel, K. Narasimharao, C.W. Macosko, K.A. Mkhoyan, S. Al-Thabaiti, B. Stottrup, M. Tsapatsis, 2D Zeolite Coatings: Langmuir-Schaefer Deposition of $3 \mathrm{~nm}$ Thick MFI Zeolite Nanosheets, Angewandte Chemie International Edition, 54 (2015) 6571-6575.

[11] G. Luo, A.G. McDonald, Conversion of Methanol and Glycerol into Gasoline via ZSM-5 Catalysis, Energy \& Fuels, 28 (2014) 600-606.

[12] M. Stöcker, Methanol-to-hydrocarbons: catalytic materials and their behavior1, Microporous and Mesoporous Materials, 29 (1999) 3-48.

[13] N.B. Milestone, D.M. Bibby, Adsorption of alcohols from aqueous solution by ZSM-5, Journal of Chemical Technology and Biotechnology. Chemical Technology, 34 (1984) 73-79.

[14] N.B. Milestone, D.M. Bibby, Concentration of alcohols by adsorption on silicalite, Journal of Chemical Technology and Biotechnology, 31 (1981) 732-736.

[15] B. Cekova, D. Kocev, E. Kolcakovska, D. Stojanova, Zeolites as alcohol adsorbents from aqueous solutions, Acta Period. Technol., (2006).

[16] M.M. Dubinin, G.U. Rakhmatkariev, A.A. Isirikyan, Differential heats of adsorption and adsorption isotherms of alcohols on silicalite, Bulletin of the Academy of Sciences of the USSR, Division of chemical science, 38 (1989) 1950-1953.

[17] P. Kumar, K.V. Agrawal, M. Tsapatsis, K.A. Mkhoyan, Quantification of thickness and wrinkling of exfoliated two-dimensional zeolite nanosheets, Nat Commun, 6 (2015).

[18] J. Kestell, e. al., A stand-alone polarization-modulation instrument optimized for the study of catalytic processes at elevated pressures, Rev. Sci. Instrum., (2016) In preparation. . 
[19] J.-Q. Zhong, J. Kestell, I. Waluyo, S. Wilkins, C. Mazzoli, A. Barbour, K. Kaznatcheev, M. Shete, M. Tsapatsis, J.A. Boscoboinik, Oxidation and Reduction under Cover: Chemistry at the Confined Space between Ultrathin Nanoporous Silicates and Ru(0001), The Journal of Physical Chemistry C, 120 (2016) 8240-8245. [20] E.M. Flanigen, H. Khatami, H.A. Szymanski, Infrared structural studies of zeolite frameworks, Adv. Chem. Ser.1971, pp. 201.

[21] A. Zecchina, S. Bordiga, G. Spoto, L. Marchese, G. Petrini, G. Leofanti, M. Padovan, Silicalite characterization. 1. Structure, adsorptive capacity, and IR spectroscopy of the framework and hydroxyl modes, The Journal of Physical Chemistry, 96 (1992) 4985-4990.

[22] N. Zvereva-Loëte, A. Ballandras, G. Weber, M. Rotger, V. Boudon, J.M. Simon, Experimental IR study and ab initio modelling of ethylene adsorption in a MFI-type host zeolite, Molecular Physics, 107 (2009) 2081-2093.

[23] V.C. Farmer, J.D. Russell, The infra-red spectra of layer silicates, Spectrochimica Acta, 20 (1964) 1149-1173.

[24] I. Doroshenko, V. Pogorelov, V. Sablinskas, Infrared Absorption Spectra of Monohydric Alcohols, Dataset Papers in Chemistry, 2013 (2013) 6.

[25] B.-T. Lonstad Bleken, L. Mino, F. Giordanino, P. Beato, S. Svelle, K.P. Lillerud, S. Bordiga, Probing the surface of nanosheet H-ZSM-5 with FTIR spectroscopy, Physical Chemistry Chemical Physics, 15 (2013) 13363-13370.

[26] K. Na, M. Choi, W. Park, Y. Sakamoto, O. Terasaki, R. Ryoo, Pillared MFI Zeolite Nanosheets of a Single-Unit-Cell Thickness, Journal of the American Chemical Society, 132 (2010) 4169-4177.

[27] T. Shimanouchi, Tables of Molecular Vibrational Frequencies Consolidated Volume I, National Bureau of Standards., (1972) 1-160.

[28] P. Bai, M. Tsapatsis, J.I. Siepmann, Multicomponent Adsorption of Alcohols onto Silicalite-1 from Aqueous Solution: Isotherms, Structural Analysis, and Assessment of Ideal Adsorbed Solution Theory, Langmuir, 28 (2012) 15566-15576.

[29] R. Xiong, S.I. Sandler, D.G. Vlachos, Molecular Screening of Alcohol and Polyol Adsorption onto MFI-Type Zeolites, Langmuir, 28 (2012) 4491-4499.

[30] R. Xiong, S.I. Sandler, D.G. Vlachos, Alcohol Adsorption onto Silicalite from Aqueous Solution, The Journal of Physical Chemistry C, 115 (2011) 18659-18669.

[31] S. Musi , N. Filipovi -Vincekovi , L. Sekovani Precipitation of amorphous $\mathrm{SiO} 2$ particles and their properties, Brazilian Journal of Chemical Engineering, 28 (2011) 89-94.

[32] R. Ravishankar, C. Kirschhock, B.J. Schoeman, P. Vanoppen, P.J. Grobet, S. Storck, W.F. Maier, J.A. Martens, F.C. De Schryver, P.A. Jacobs, Physicochemical Characterization of Silicalite-1 Nanophase Material, The Journal of Physical Chemistry B, 102 (1998) 2633-2639.

[33] R. Włodarczyk, M. Sierka, J. Sauer, D. Löffler, J. Uhlrich, X. Yu, B. Yang, I. Groot, S. Shaikhutdinov, H.-J. Freund, Tuning the electronic structure of ultrathin crystalline silica films on $\mathrm{Ru}(0001)$, Physical Review B, 85 (2012).

[34] D. Loffler, J.J. Uhlrich, M. Baron, B. Yang, X. Yu, L. Lichtenstein, L. Heinke, C. Buchner, M. Heyde, S. Shaikhutdinov, H.J. Freund, R. Wlodarczyk, M. Sierka, J. 
Sauer, Growth and Structure of Crystalline Silica Sheet on $\mathrm{Ru}(0001)$, Physical Review Letters, 105 (2010) 4.

[35] M. Wang, J. Zhong, J.D. Kestell, I. Waluyo, D. Stacchiola, J.A. Boscoboinik, D. $\mathrm{Lu}$, Surface and interfacial dipoles driven energy level shift in the silica/Ru(0001) heterojunction, (Submitted to Topics in Catalysis). 


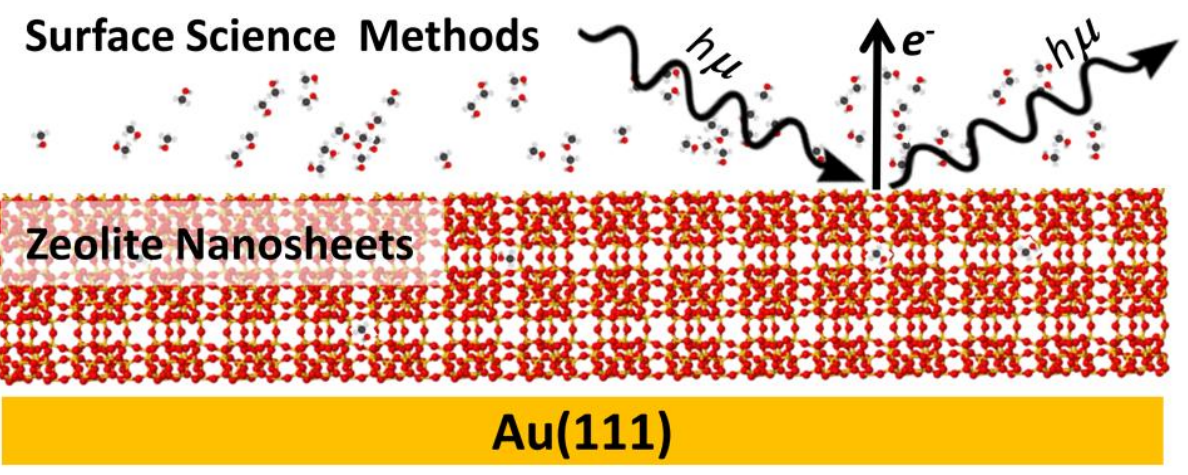

16 